\title{
Multiple Hydrogen Bonds Tuning Guest/Host Excited-State Proton Transfer Reaction: Its Application in Molecular Recognition
}

\author{
He-Chun Chou, ${ }^{\dagger}$ Chin-Hao Hsu, ${ }^{\ddagger}$ Yi-Ming Cheng, ${ }^{\dagger}$ Chung-Chih Cheng, ${ }^{\dagger}$ Hsiao-Wei Liu,${ }^{\dagger}$ \\ Shih-Chieh Pu, ${ }^{\dagger}$ and Pi-Tai Chou*, ${ }^{*}$ \\ Department of Chemistry, National Taiwan University, No. 1, Sec. 4, Roosevelt Road, Taipei, Taiwan, R.O.C, and \\ Department of Chemistry and Biochemistry, National Chung Cheng University, Chia-Yi, Taiwan, R.O.C.
}

Received October 27, 2003; E-mail: chop@ntu.edu.tw

Among various types of approaches for biorecognition, the principle of using hydrogen bonds to confer binding strength and selectivity is of particular interest ${ }^{1 \mathrm{a}-\mathrm{g}}$ mainly due to their relative flexibility in geometry compared to rigid covalent bonds. ${ }^{1 \mathrm{~h}, \mathrm{i}}$ Because guest molecules of biological interest may possess various numbers of proton-donating and/or -accepting groups, the design and syntheses of host receptors providing multiple hydrogen-bonding sites are necessary to maximize the recognition capacity. We further proposed that induced by the multiple hydrogen bonds, the redistribution of $\pi$ electrons might result in unusual photophysical properties such as the host/guest type of excited-state proton-transfer (ESPT) reaction, ${ }^{2}$ providing a superb opportunity for enhancing signal transduction.

In an effort to apply this concept to molecular recognition, we have examined numerous multiple-hydrogen-bonding (HB) systems, among which 3,4,5,6-tetrahydrobis(pyrido[3,2-g]indolo)[2,3-a:3', 2'j] acridine (1a, Scheme 1), designed and synthesized by Thummel and co-workers, ${ }^{3}$ is an exquisite case in point. 1a was designed so that pyrrole and pyridine moieties function as the proton donor and acceptor, respectively. Two symmetric push-pull conjugated units form a V-shaped framework with a cleft of appropriate size to provide as many as five HB sites. This, in combination with a flexible dimethylene skeleton, renders a preorganized motif particularly suitable for the multiple-HB recognition. The HB association between 1a and urea derivatives has been investigated via monitoring of the ${ }^{1} \mathrm{H}$ NMR chemical shifts. ${ }^{3}$. We report here certain previously unrecognized, remarkable photophysical properties in that the catalytic versus noncatalytic 1a HB systems (vide infra) play a key role in tuning ESPT, which leads to a feasible design for sensing multiple-HB-site analytes of biological interest.

Upon titration of $\mathbf{1 a}$ with acetic acid, the $405 \mathrm{~nm}$ vibronic band ascribed to the $S_{0}-S_{1}\left(\pi \pi^{*}\right)$ transition of free $\mathbf{1 a}$ in benzene revealed bathochromic shifts with the appearance of an isosbestic point at $410 \mathrm{~nm}$ (Figure 1). The measured absorbance $\left[A_{0} /(A-\right.$ $\left.A_{0}\right)$ ] as a function of [acetic acid $]^{-1}$ fit a linear relationship, ${ }^{4}$ supporting the 1:1 HB complex formation. The ratio for the intercept versus slope deduced a $K_{\mathrm{a}}$ value of $(8.0 \pm 0.5) \times 10^{3} \mathrm{M}^{-1}$ (see Supporting Information). During the titration, drastic quenching of the $420 \mathrm{~nm}$ fluorescence intensity was observed, accompanied by the appearance of a weak, large Stokes shifted emission maximized at $\sim 600 \mathrm{~nm}$ and an isoemissive point at $580 \mathrm{~nm}$ (see insert of Figure 1). A linear plot of $F_{0} /\left(F-F_{0}\right)$ versus [acetic acid] $]^{-1}$ for both bands reconfirmed the $1: 1$ complex formation, and $K_{\mathrm{a}}$ was deduced to be $(8.3 \pm 0.3) \times 10^{3} \mathrm{M}^{-1}$ (see Supporting Information). ${ }^{4}$ The excitation spectrum monitored at the $420 \mathrm{~nm}$ band is identical to the absorption profile of free 1a, while it is red shifted with respect to that of the $420 \mathrm{~nm}$ band upon being monitored at the $600 \mathrm{~nm}$

\footnotetext{
* To whom correspondence should be addressed.

National Taiwan University.

\$ National Chung Cheng University.
}

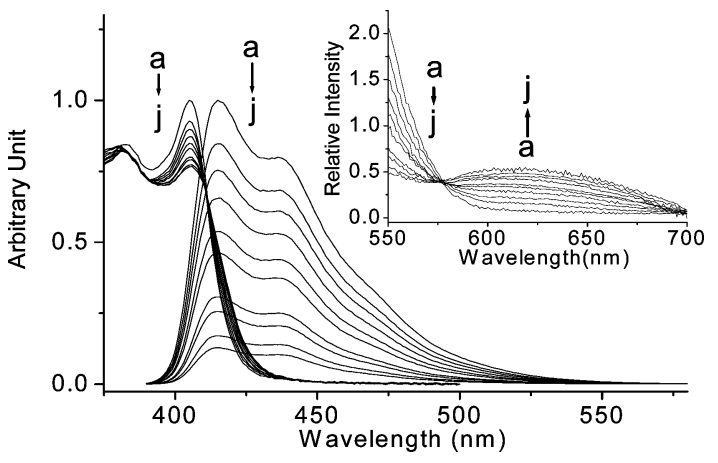

Figure 1. Absorption and emission of $1 \mathrm{a}\left(1.2 \times 10^{-5} \mathrm{M}\right)$ in benzene by adding [acetic acid] of (a) 0, (b) 1, (c) 2, (d) 4, (e) 6, (f) 10, (g) 20, (h) 40, (i) 50 , (j) 80 equiv $\left(1\right.$ equiv $\left.=1.5 \times 10^{-5} \mathrm{M}\right)$. Insert: Enlargement of fluorescence titration at $>550 \mathrm{~nm}$.

Scheme 1. Optimized Structures of Various 1a/Guest Complexes Calculated by PM3 Method

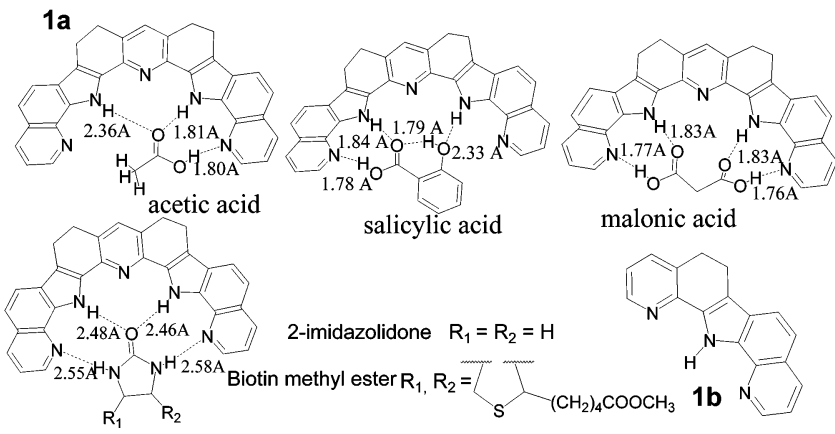

band (see Supporting Information). These results, in combination with significantly different lifetimes between $420 \mathrm{~nm}(1.60 \mathrm{~ns})$ and $600 \mathrm{~nm}(0.24 \mathrm{~ns})$ bands, led us to conclude that the dual fluorescence originates from different ground-state precursors, namely, the uncomplexed 1a and 1:1 1a/acetic acid HB complex. ESPT takes place in the 1:1 $\mathbf{1 a}$ /acetic acid HB complex (Scheme 2), resulting in an $\sim 7200 \mathrm{~cm}^{-1}$ Stokes shifted imino-like tautomer emission. A further approach using the femtosecond fluorescence upconversion technique (see Supporting Information) revealed a system-response limited ( $<150 \mathrm{fs}$ ) rise component at the $600 \mathrm{~nm}$ band, indicating the occurrence of an ultrafast, possibly barrierless ESPT reaction in the 1a/acetic acid HB complex.

Other biosignificant carboxylic acids possessing multiple $\mathrm{HB}$ sites such as malonic acid and salicylic acid (see Scheme 1) were also investigated. From the absorption titration, $K_{\mathrm{a}}$ values were deduced to be $(1.1 \pm 0.1) \times 10^{5}$ and $(5.4 \pm 0.2) \times 10^{3} \mathrm{M}^{-1}$ for $1 \mathrm{a} / \mathrm{malonic}$ acid and 1a/salicylic acid HB complexes, respectively. For both systems, similar to the case of $\mathbf{1 a / a c e t i c ~ a c i d , ~ t h e ~ d e c r e a s e ~ o f ~} 420$ $\mathrm{nm}$ fluorescence accompanied by the appearance of a weak, $\sim 600$ $\mathrm{nm}$ emission was observed during the titration, ${ }^{5}$ supporting the 


\section{Scheme 2}
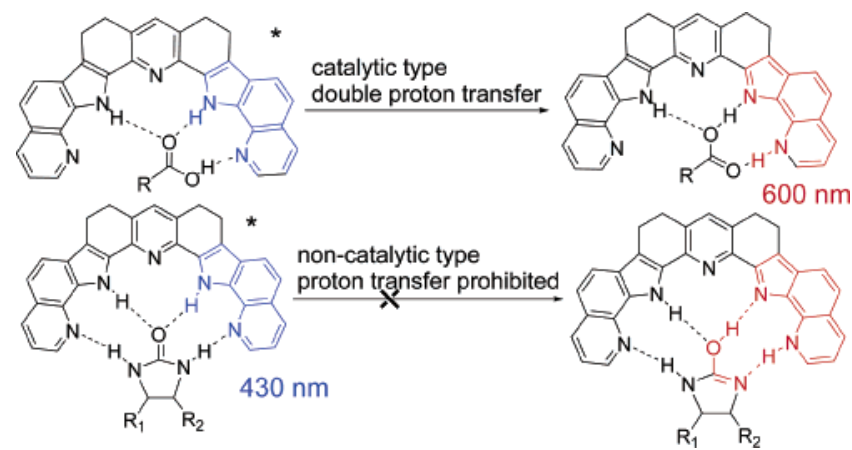

occurrence of an ESPT reaction through the HB complex formation. Although attempts to grow single crystals of corresponding 1a/carboxylic acids HB complexes were not successful, their structure might qualitatively be rationalized by the molecular modeling, ${ }^{6}$ in which the 1a/acetic acid complex revealed a stable complexation incorporating triple hydrogen bonds, while a well-fitted quadruple HB 1a/malonic acid complex was resolved (see Scheme 1). For the case of the 1a/salicylic acid triple-HB complex, the $K_{\text {a }}$ value smaller than that of $\mathbf{1 a}$ /acetic acid is possibly due to the preorganization of the phenyl moiety in fitting the cleft of 1a (see Scheme 1). Support for the multiple HB formation is also given by the synthesis of 6,13-dihydro-5H-1,12,13-triaza-dibenzo[a,i]fluorene (1b, Scheme 1; Supporting Information), which is considered as a half unit of 1a, so that an upper limit of dual hydrogen bonds can be formed with acetic acid. Although the formation of $\mathbf{1 b}$ /acetic acid hydrogen-bonded complex was observed as well, a much smaller association constant of $(5.8 \pm 0.2) \times 10^{2} \mathrm{M}^{-1}$ was deduced in benzene.

Upon addition of urea derivatives such as 2-imidazolidone and biotin methyl ester (BME see Scheme 1), the absorption titration revealed spectral red shifts similar to those of $\mathbf{1 a}$ /carboxylic acids. However, during the fluorescence titration, instead of the quenching of $420 \mathrm{~nm}$ emission, as in the case of carboxylic acids due to ESPT, the $420 \mathrm{~nm}$ fluorescence exhibits a bathochromic shift throughout the titration (Figure 2). The appearance of an isoemissive point at $\sim 425 \mathrm{~nm}$ with a dual lifetime $\left(\tau_{\mathrm{f}} \sim 1.60\right.$ and $\left.3.61 \mathrm{~ns}\right)$, in combination with a straight line for the plot of $\left[F_{0} /\left(F-F_{0}\right)\right]$ versus [imidazolidone] $^{-1}$ (see insert of Figure 2), is consistent with the 1:1 1a/imidazolidone (or 1a/BME) HB complexation. Accordingly,

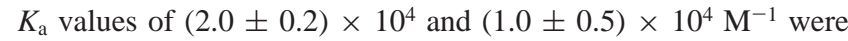
deduced for $1 \mathbf{a} /$ imidazolidone and $1 \mathbf{a} / \mathrm{BME}$, respectively. The large association constants can be qualitatively rationalized by the quadruple HB 1a/imidazolidone complex resolved from X-ray structure $^{3}$ as well as modeling (Scheme 1).

Accordingly, depending on the properties of the guest molecules, the photophysics of the 1a/guest complex vary drastically. On the basis of the chemical aspects of guest-molecule-assisted ESPT, we can classify the 1a/guest HB complexes into two categories. As depicted in Scheme 2, the carboxylic acid assisted ESPT in 1a can be specified as a catalytic process because following the ESPT reaction the molecular structure of the carboxylic functional group remains unchanged. Conversely, both $\mathbf{1 a}$ and urea derivatives such as 2-imidazolidone and BME would have tautomerized (i.e., lactam $\rightarrow$ lactim) simultaneously if ESPT in 1a/ureas HB complexes had taken place. This type of reaction is defined as a noncatalytic process, in which ESPT depends not only on the host (i.e., the receptor) but also on the isomerization of the guest molecule (i.e., the analyte) and is thus expected to be energetically less favorable. A qualitative approach from the PM3 method indicates that catalytic ESPT in 1a/acetic acid is thermodynamically allowed by $\sim 3 \mathrm{kcal} /$

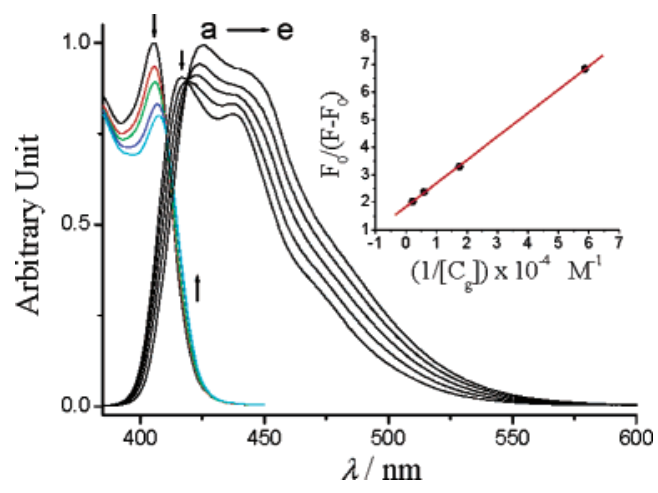

Figure 2. Fluorescence titration spectra of $1 \mathrm{a}\left(1.2 \times 1 \overline{0}^{-5} \mathrm{M}\right)$ in benzene upon adding 2-imidazolidone of (a) 0, (b) 3, (c) 10, (d) 30, (e) 80 equiv (1 equiv $\left.=5.7 \times 10^{-6} \mathrm{M}\right)$. Insert: Plot of $F_{0} /\left(F-F_{0}\right)$ at $475 \mathrm{~nm}$ versus

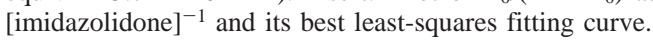

mol. In contrast, it is prohibited in the case of the noncatalytic 1a/2-imidazolidone HB complex due to the $\sim 4 \mathrm{kcal} / \mathrm{mol}$ endergonic energy required for simultaneous tautomerization of $\mathbf{1 a}$ and 2-imidazolidone (see Supporting Information).

In conclusion, we present a recognition concept utilizing multiplehydrogen-bond fine-tuned excited-state double-proton-transfer reaction. The catalytic versus noncatalytic ESPT demonstrates its suitability in differentiating carboxylic acids and urea derivatives. Although current applications of $\mathbf{1 a}$ are limited in organic solvents, future conceptual design may focus on water-soluble multiple-HB receptors, in which the $\mathrm{V}$-shape cleft allows only a few water molecules to be accommodated so that the multiple-HB strength for substrates of interest is competitively strong. In the case of $\mathbf{1 a}$, ESPT was observed by addition of $\mathrm{H}_{2} \mathrm{O}\left(>10^{-4} \mathrm{M}\right)$ in $\mathrm{CH}_{3} \mathrm{CN}$. The occurrence of ESPT in protic solvents has the advantage of selectively probing urea derivatives due to the prohibition of ESPT in 1a/ureas complexes. The enhancement of normal emission may thus be exploited as the signal transduction. Syntheses focusing on the multiple-hydrogen-bond-coupled ESIPT reaction in aqueous solution are currently in progress.

Supporting Information Available: Detailed experimental procedures and absorption, emission, time-resolved and X-ray studies. This material is available free of charge via the Internet at http://pubs.acs.org.

\section{References}

(1) (a) Davis, A. P.; Wareham, R. S. Angew. Chem., Int. Ed. 1999, 38, 2978. (b) Mizutani, T.; Kurahashi, K.; Murakami, T.; Matsumi, N.; Ogoshi, H. J. Am. Chem. Soc. 1997, 119, 8991. (c) Davis, A. P.; Wareham, R. S Angew. Chem., Int. Ed. 1998, 37, 2270. (d) Inouye, M.; Takahashi, K.; Nakazumi, H. J. Am. Chem. Soc. 1999, 121, 341. (e) Mazik, M.; Sicking, W. Chem.-Eur. J. 2001, 7, 664. (f) Yoshimoto, K.; Nishizawa, S.; Minagawa, M.; Teramae, N. J. Am Chem. Soc. 2003, 125, 8982. (g) Chin J.; Chung, S.; Kim, D. H. J. Am. Chem. Soc. 2002, 124, 10948. (h) Yang, W.; He, H.; Drueckhammer, D. G. Angew. Chem., Int. Ed. 2001, 40, 1714 (i) Ajayaghosh, A.; Arunkumar, E.; Daub, J. Angew. Chem., Int. Ed. 2002 $41,1766$.

(2) Chou, P. T.; Wei, C. Y.; Wu, G. R.; Chen, W. S. J. Am. Chem. Soc. 1999, $121,12186$.

(3) (a) Hegde, V.; Madhukar, P.; Madura, J. D.; Thummel, R. P. J. Am. Chem. Soc. 1990, 112, 4549. (b) Hedge, V.; Hung, C. Y.; Madhukar, P.; Cunningham, R.; Hopfner, T.; Thummel, R. P. J. Am. Chem. Soc. 1993 115,872 .

(4) $A_{0}$ and $A$ are the absorbance of free 1a and the measured absorbance during the titration, respectively. Similarly, $F_{0}$ and $F$ denote the fluorescence intensity of free and titrant added 1a. See Supporting Information for details.

(5) Noted that upon adding higher concentrations $\left(>10^{-3} \mathrm{M}\right)$ of malonic acid or salicylic acid protonation takes place, possibly due to increases in the local polarity, resulting in 1a cationic emission maximum at $\sim 530 \mathrm{~nm}$.

(6) Molecular modeling was performed by the semiempirical PM3 method using a Spartan program package (release 3.1.6, Wavefunction, Inc., Irvine, 1994).

JA039240F 